\title{
MicroRNA-3148 acts as molecular switch promoting malignant transformation and adipocytic differentiation of immortalized human bone marrow stromal cells via direct targeting of the SMAD2/TGF $\beta$ pathway
}

\author{
Radhakrishnan Vishnubalaji', Ramesh Elango ${ }^{1}$, Muthurangan Manikandan², Abdul-Aziz Siyal', Dalia Ali ${ }^{3}$,
} Ammar Al-Rikabi', Dana Hamam, Rimi Hamam ${ }^{6}$, Hicham Benabdelkamel ${ }^{7}$, Afshan Masood ${ }^{7}$, Ibrahim O. Alanazi ${ }^{8}$, Assim A. Alfadda7, Musaad Alfayez ${ }^{2}$, Abdullah Aldahmash², Moustapha Kassem²,3,9 and Nehad M. Alajez ${ }^{1}$

\begin{abstract}
MicroRNAs (miRs/miRNAs) play a key role in posttranscriptional regulation of gene expression and are implicated in a number of physiological and pathological conditions, including cellular malignant transformation. In the current study, we investigated the role of miR-3148 in regulating human stromal (mesenchymal) stem cell (hMSC) differentiation and transformation. Stable expression of miR-3148 in telomerized hMSC (hMSC-miR-3148) led to significant increase in in vitro adipocytic differentiation and suppression of osteoblastic differentiation. Concordantly, global gene expression profiling revealed significant enrichment in cholesterol biosynthesis pathway, and pathways related to enhanced cell movement and survival, whereas processes related to bone and connective tissue developments, cell death, apoptosis, and necrosis were downregulated. Global proteomic analysis using 2D-DIGE followed by mass spectrometry (MS) revealed significant changes in protein expression in hMSC-miR-3148 and enrichment in protein networks associated with carcinogenesis. Functional studies revealed that hMSC-miR-3148 exhibited enhanced in vitro cell proliferation, colony formation, migration, invasion, sphere formation, doxorubicin resistance, and increased active number of cells in $S$ and $G 2 / M$ cell cycle phases and formed sarcoma-like tumors with adipocyte infiltration when implanted into immunocompromised mice. SMAD2 was identified as bone fide gene target for miR-3148 using qRT-PCR, Western blotting, and UTR-based reporter assay. In agreement with our data, SMAD2 expression was downregulated in 47\% of patients with soft tissue sarcoma. Bioinformatics analysis revealed that elevated miR-3148 expression correlates with poor prognosis in several human cancer types, including sarcoma. Our study identified miR-3148 as factor regulating hMSC differentiation and is involved in promoting malignant transformation of telomerized hMSC.
\end{abstract}

Correspondence: Nehad M. Alajez (nalajez@hbku.edu.qa)

'Cancer Research Center, Qatar Biomedical Research Institute (QBRI), Hamad Bin Khalifa University (HBKU), Qatar Foundation (QF), Doha, Qatar

${ }^{2}$ Stem Cell Unit, Department of Anatomy, College of Medicine, King Saud

University, Riyadh, Saudi Arabia

Full list of author information is available at the end of the article

Edited by N. Barlev

\section{Introduction}

MicroRNAs (miR/miRNA) are regulatory noncoding double-stranded RNAs that exert posttranscriptional regulation of gene expression under normal and pathological conditions ${ }^{1}$. Global transcriptomic analysis of miRNAs in cancer tissue compared with normal tissue revealed differential expression of a number of miRNAs which are either downregulated in cancer and thus exert a

\section{(-) The Author(s) 2020}

(c) (i) Open Access This article is licensed under a Creative Commons Attribution 4.0 International License, which permits use, sharing, adaptation, distribution and reproduction in any medium or format, as long as you give appropriate credit to the original author(s) and the source, provide a link to the Creative Commons license, and indicate changes were made. The images or other third party material in this article are included in the article's Creative Commons license, unless indicated otherwise in a credit line to the material. If material is not included in the article's Creative Commons license and your intended use is not permitted by statutory regulation or exceeds the permitted use, you will need to obtain permission directly from the copyright holder. To view a copy of this license, visit http://creativecommons.org/licenses/by/4.0/. 
tumor suppressor role through direct targeting of oncogenes or upregulated miRNAs, which act as oncomiRs through inhibition of tumor suppressor genes ${ }^{2}$. Deregulated miRNA expression is associated with large number of human cancers, including breast, colon, gastric, lung, and sarcomas ${ }^{3-8}$. MiRNAs have also been implicated in regulating epithelial-to-mesenchymal transition, a process leading to endowment of cancer cells with a mesenchymal phenotype?

MiRNAs have also been implicated in regulating stem cell differentiation ${ }^{10}$. For instance, we have previously shown the miR-320 family to promote adipocytic and to inhibit osteoblastic differentiation of human bone marrow stromal (mesenchymal) stem cells (hBMSCs) through repression of RUNX2 expression ${ }^{11}$. Interestingly, we also identified the miR-320 family to suppress colorectal cancer, through direct targeting of FOXM1, FOXQ1, and the stem cell marker, SOX4 ${ }^{12}$, suggesting that miRNAs that play a role in regulating stem cell functions, may be associated with cancer development ${ }^{13}$.

In the current study, we investigated the biological effects of miR-3148, identified from our previous microRNA profiling during hMSC differentiation, on hMSC cell differentiation into osteoblast and adipocytes. Functional and molecular investigations revealed miR-3148 to promote adipogenesis and to suppress osteogenesis through direct targeting of SMAD2/TGF $\beta$ pathway. In addition, hMSC-miR-3148 exhibited enhanced in vitro cell proliferation, colony formation, cell migration, cell invasion and sphere formation, increased active $\mathrm{S}$ and G2/M cell cycle phases, and formed sarcoma-like tumors when implanted into immunocompromised mice, suggesting a role for miR-3148 as regulator of stem cell differentiation and transformation.

\section{Results}

\section{MiR-3148 overexpression enhances adipocytic and} suppresses osteoblastic differentiation

Our previous global miRNA profiling identified miR3148 as significantly downregulated miRNA during osteoblastic differentiation of human stromal (mesenchymal) stem cells (Supplementary Fig. 1). To unravel potential role for miR-3148 during stem cell differentiation, hMSC cells were transduced with a lentiviral vector encoding for miR-3148 (hMSC-miR-3148) which exhibited significant upregulation of miR-3148 expression compared to hMSC-control cells (Supplementary Fig. 2). Subsequently, hMSC-miR-3148 and hMSC-control cells were subjected to adipocytic and osteoblastic differentiation conditions. An enhanced adipocytic differentiation was observed in hMSC-miR-3148 as evidenced by oil red staining (Fig. 1a), Nile red quantification (Fig. 1b), and quantitative reverse transcriptase-polymerase chain reaction (qRT-PCR) for adipocyte protein 2 (AP2) and leptin
(LEP, Fig. 1c). On the other hand, hMSC-miR-3148 exhibited diminished ALP activity (Fig. 1d, e), decreased in vitro mineralization (Fig. 1f, g) and decreased expression of osteoblastic gene markers: osteocalcin (OC), osteonectin (ON), alkaline phosphatase (ALP), and Runtrelated transcription factor 2 (RUNX2, Fig. 1h).

\section{miR-3148 regulates multiple genetic pathways in hMSC}

To gain more insight into the molecular mechanisms by which miR-3148 exert its effects during hMSC differentiation, we performed global transcriptome analysis of hMSC-miR-3148 compared to hMSC-control cells and identified 1476 upregulated and 2370 downregulated genes (2.0-fold change, $p<0.05$, Supplementary Table 1 ) in the hMSC-miR-3148. Hierarchical clustering showed clear separate clustering of the hMSC-miR-3148 and hMSC-control cells (Fig. 2a). The list of upregulated genes were subsequently subjected to Ingenuity ${ }^{\circledR}$ Pathway Analysis (IPA). Data presented in Fig. $2 \mathrm{~b}$ depict the top five enriched canonical pathways enriched in hMSC-miR3148 cells and included cholesterol biosynthesis. Analysis for functional categories revealed significant enrichment in several categories, including cellular movement, cellular invasion, and cellular survival, while categories related to cell death, apoptosis, and necrosis were significantly underrepresented (Supplementary Fig. 3). The cellular movement and cellular death and survival functional categories are illustrated in Supplementary Fig. 3 as well. IPA analysis on the downregulated genes in hMSCmiR-3148 cells reveled significant downregulation in tissue development functional categories (Supplementary Fig. 3). Upstream regulator analysis of the differentially expressed genes revealed significant enrichment in a number of networks driven by Mitogen-Activated Protein Kinase 1 (MAPK1), Interleukin 1 Receptor Antagonist, Triggering Receptor Expressed On Myeloid Cells 1, Activating Transcription Factor 4, Bruton Tyrosine Kinase, TGF-Beta Activated Kinase 1 (MAP3K7) Binding Protein 1 (TAB1), Amphiregulin (AREG), CCR4-NOT Transcription Complex Subunit 7 (CNOT7), Vascular Endothelial Growth Factor A, and TAL BHLH Transcription Factor 1, Erythroid Differentiation Factor (TAL1) (Supplementary Fig. 3).

In addition, we performed global proteomic analysis using 2D-DIGE followed by protein identification by mass spectrometry (MS). Proteomic analysis identified 2000 spots consistently mapped to all gels (Fig. 2c). Sixty protein spots exhibited statistically significant change in abundance in hMSC-miR-3148 compared to hMSCcontrol cells (ANOVA-test $p<0.05$; fold change $>1.5$ ). These spots were picked and further analyzed by MALDITOF MS and identified 40 spots, which were matched using the MASCOT peptide mass fingerprints (PMF) to entries in the SWISS-PROT database with high 


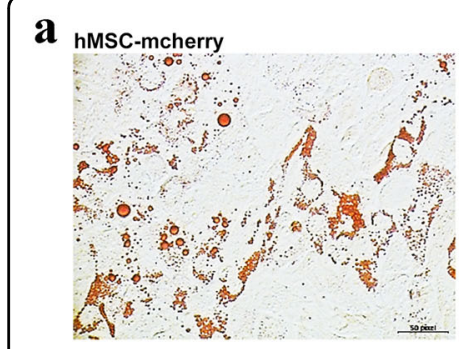

d

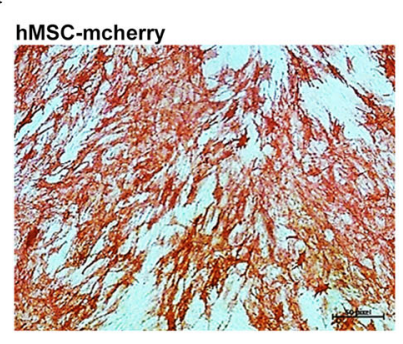

g

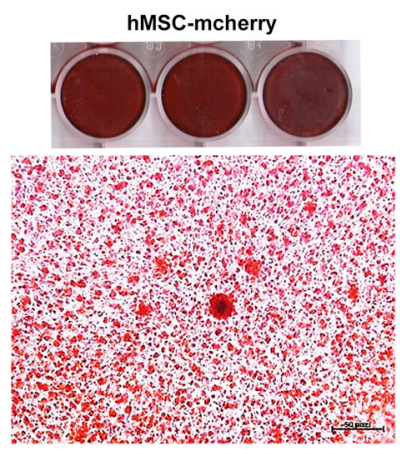

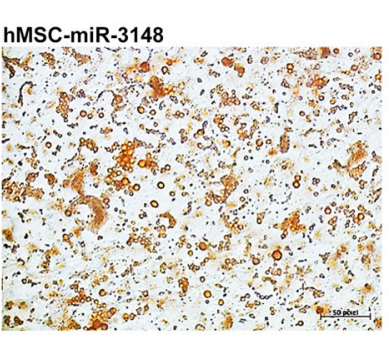
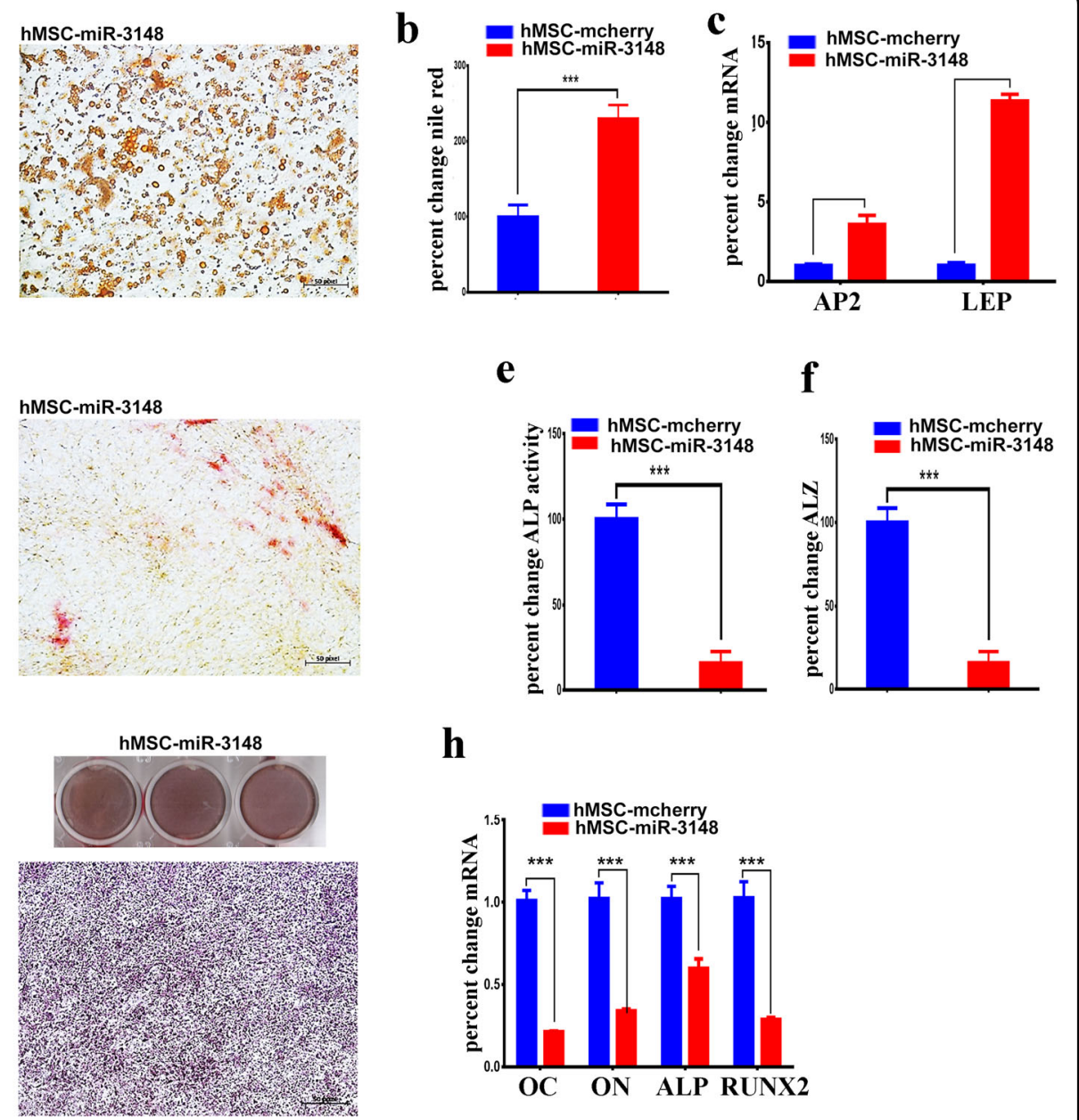

e

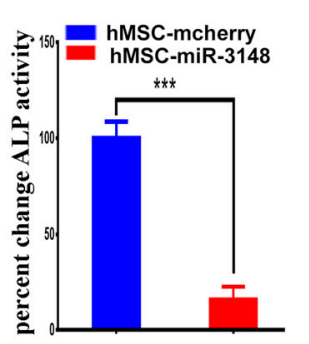

h

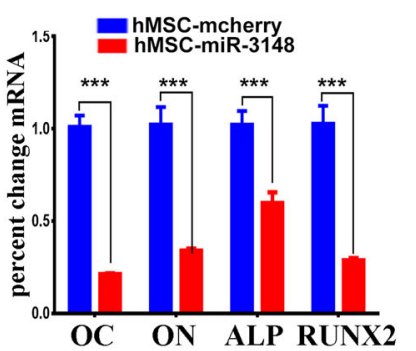

Fig. 1 hMSC-miR-3148 cells exhibited enhanced adipocytic and reduced osteoblastic differentiation of hMSC. a Representative Oil Red-O staining on day 7 showing increased adipogenesis in hMSC-miR-3148 cells. Quantification of nile red staining (b) and adipocyte-specific gene marker (AP2 and LEP) expression (c) in hMSC-miR-3148 vs hMSC-mcherry cells. d Representative ALP staining of osteoblast cells on day 10 in hMSC-miR-3148 vs hMSC-mcherry cells. Quantification of ALP activity (e) and alizarin red (ALZ, $\mathbf{f})$ in hMSC-miR-3148 vs hMSC-mcherry cells. $\mathbf{g}$ Representative images of alizarin red staining of mineralized matrix in hMSC-miR-3148 vs hMSC-mcherry cells performed on day 21. $\mathbf{h}$ Quantification of osteoblast specific gene markers (OC, ON, ALP, and RUNX2) in hMSC-miR-3148 compared to hMSC-mcherry using qRT-PCR. Data are presented as mean \pm S.E.M. from three independent experiments, ${ }^{* * *} p<0.0005$.

confidence. Among the 40 spots, 21 spots were upregulated and 19 spots were downregulated in hMSC-miR3148 compared to control cells (Fig. 2c, Supplementary Fig. 4, and Supplementary Table 2). Pathway analysis of the identified proteins revealed strong enrichment in pathways associated with cancer development (Fig. 2d).

miR-3148 overexpression enhances colony formation, cell migration and invasion

Transcriptomic and proteomic data revealed enrichment in cancer-associated functional categories and signaling pathways in the hMSC-miR-3148 model. Therefore, we subsequently investigated the biological effects of forced expression of miR-3148 on hMSC colony formation, migration, and invasion. Data presented in
Fig. 3a revealed increased colony formation in hMSCmiR-3148 compared to control cells, which were observed in hMSC-miR-3148 as early as day 5 , compared to control cells. Scratch and xCELLigence real-time migration assays revealed significant increase in cell motility and migration of hMSC-miR-3148 (Fig. 3b, c). In addition, Boydenchamber transwell migration and invasion assay, revealed enhanced migration (Fig. 3d) and invasion (Fig. 3e) of hMSC-miR-3148.

\section{miR-3148 overexpression enhances cell proliferation and doxorubicin drug resistance}

hMSC-miR-3148 cells exhibited increased cell proliferation (Fig. 4a), formed a greater number of spheroids when cultured under low-adherence cell culture 


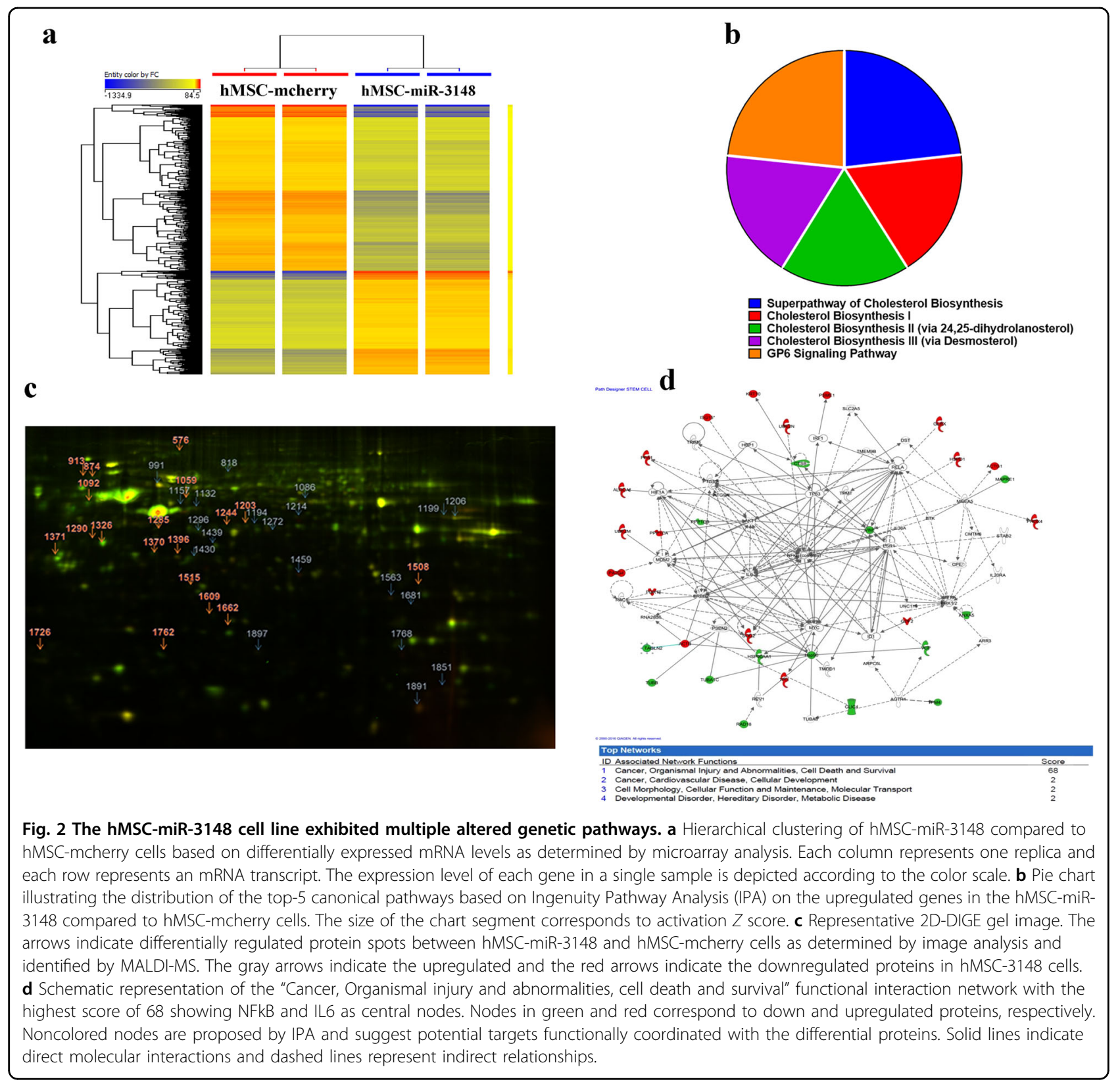

conditions (Fig. 4b). Notably, the spheroids formed by hMSC-miR-3148 were more compact whereas control cells-derived spheroids had irregular forms (Fig. 4b, zoomed). Cell cycle analysis revealed significant decrease in G0/G1 and significant increase in S and G2-M phases of the cell cycle in hMSC-miR-3148 compared to controls (Fig. 4c). Finally, hMSC-miR-3148 and control cells were exposed to doxorubicin, a commonly used chemotherapy in the treatment of human sarcomas ${ }^{14}$. hMSC-miR-3148 exhibited increased resistance to doxorubicin concentration ranging from 7.8 to $125 \mathrm{nM}$ (Fig. 4d) and formation of doxorubicin-resistant colonies was notable at 7.8 and $15.6 \mathrm{nM}$.
miR-3148 overexpression promotes sarcoma-like tumor formation in vivo

Our in vitro data demonstrated enhanced cell proliferation, colony formation, and migration in hMSC-miR3148 cells. Therefore, to examine the ability of hMSC-miR-3148 to form tumors in vivo, we implanted hMSC-miR-3148 and control cells loaded into Matrigel@ matrix, subcutaneously into immune deficient nude mice. Concordant with the in vitro data, hMSC-miR-3148 cells formed tumors, which were not detected in control cells (Fig. 5a, b). We observed sustained high expression of miR-3148 in the hMSC-miR-3148 xenografts (Fig. 5c). Histological examination of the tumor tissue revealed a 
a

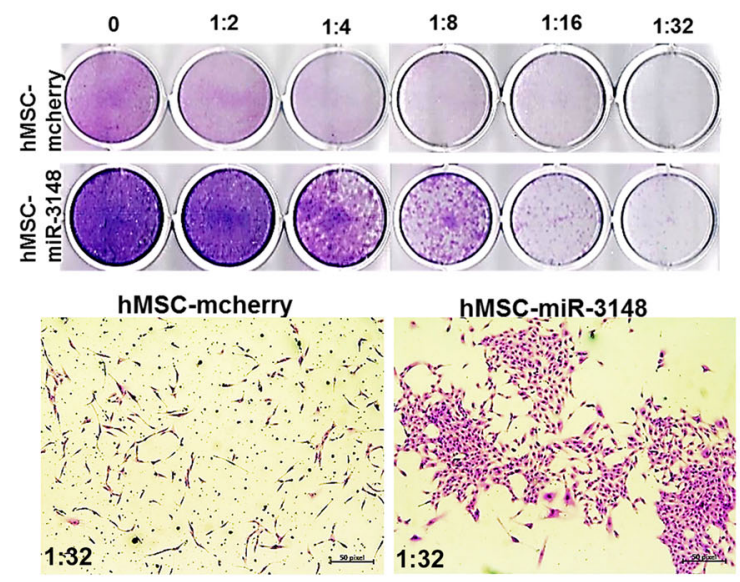

b

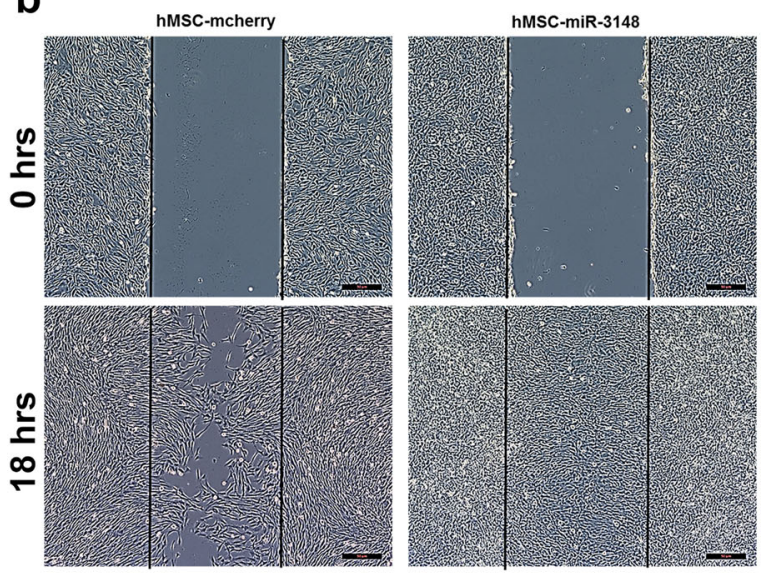

C

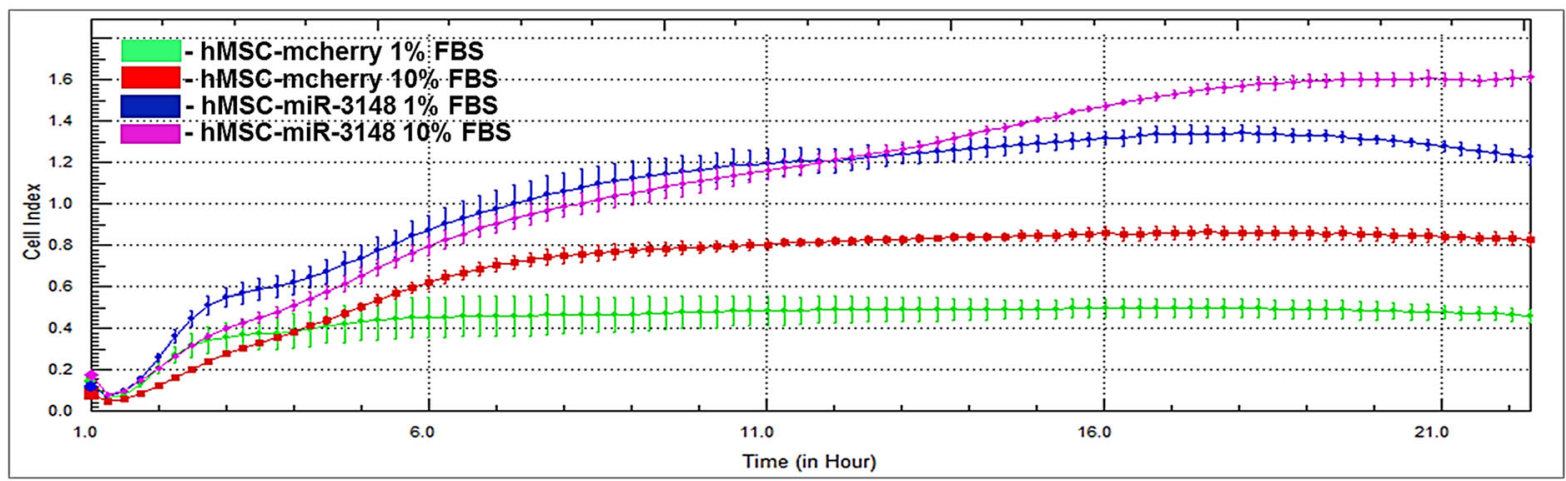

d hMSC-mcherry $1 \%$ FBS
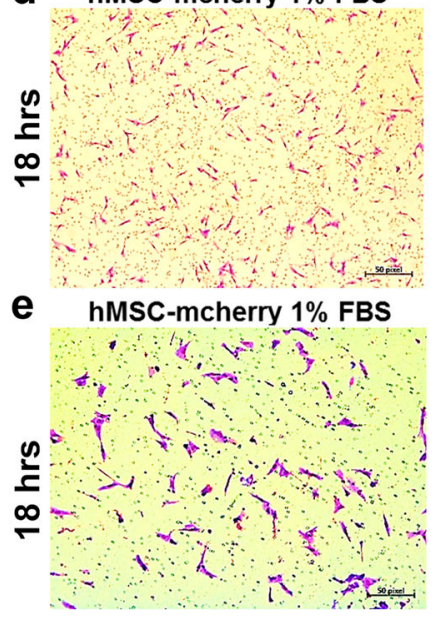
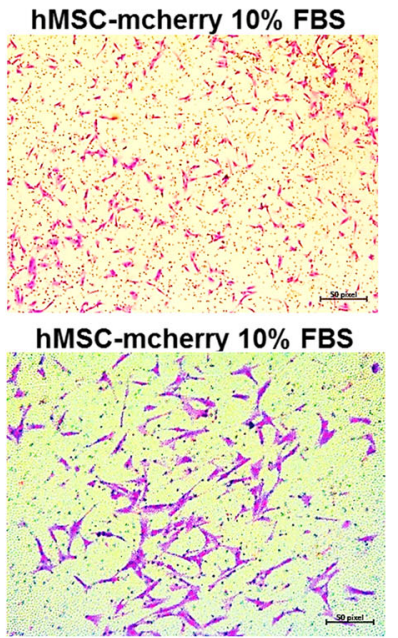
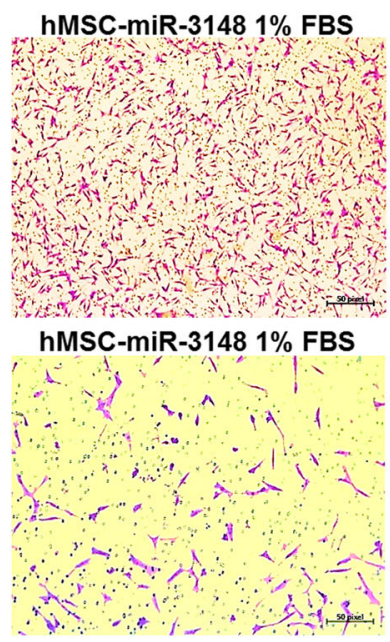

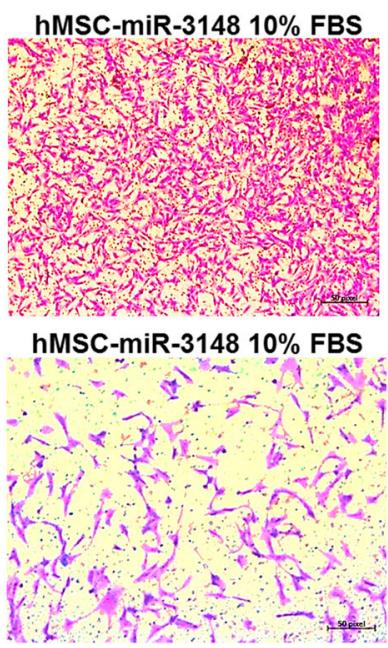

Fig. 3 The hMSC-miR-3148 cells exhibited enhanced clonogenicity, migration and invasion potential. a Clonogenic (CFU) assay showing enhanced colony forming capability of hMSC-miR-3148 compared to hMSC-mcherry control cells on day 5. Plates were stained using the Diff-Quik staining set. Data are representative of two independent experiments for each condition and representative images of formed colonies (lower panels) were taken at 1:32 dilution. b Measurement of hMSC-miR-3148 cell migration using in vitro scratch assay. Images were captures just after the injury $(0 \mathrm{~h})$ and at $24 \mathrm{~h}$. XCELLigence real-time cell analysis (RTCA) (c) and conventional (d) migration assay depicting enhanced migration of hMSCmiR-3148 cells toward 1 and 10\% serum condition compared to hMSC-mcherry control cells in a time-dependent manner. e Representative images

from conventional invasion assay demonstrating enhanced invasion capabilities hMSC-miR-3148 compared to hMSC-mcherry control cells. 


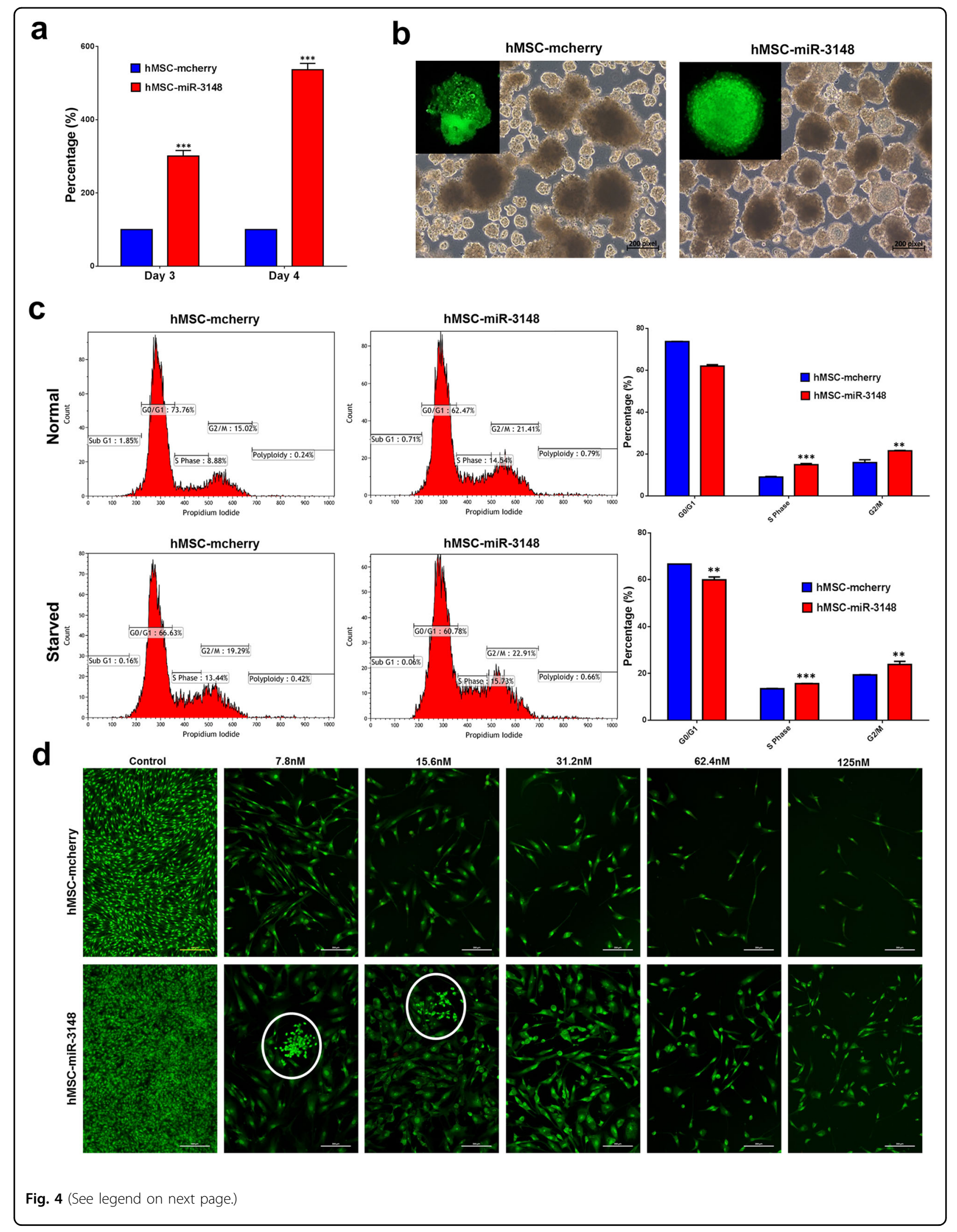


(see figure on previous page)

Fig. 4 The hMSC-miR-3148 cells exhibited enhanced cell proliferation, sphere formation, and doxorubicin resistance. a Cell proliferation measured using alamarblue assay of hMSC-miR-3148 compared to hMSC-mcherry cells on day 3 and day 4 . Data are presented as mean percent increase in cell proliferation \pm S.E.M., $n=30$ from two independent experiments, ${ }^{* *} p<0.0001$. $\mathbf{b}$ Representative images depicting sphere formation in hMSC-miR-3148 vs hMSC-mcherry cells on day 10. c Flow cytometry cell cycle analysis on hMSC-miR-3148 and hMSC-mcherry under normal (upper panels) and serum starvation (lower panels) conditions. The proportion of cells in the G0-G1, S, and G2-M phases are indicated on each plot. Quantitative analysis of cell cycle distribution in hMSC-miR-3148 and hMSC-mcherry is presented as bar diagram. Data are presented as mean \pm S.E. M., $n=3,{ }^{* *} p<0.005 ;{ }^{* * *} p<0.0005$. d Representative fluorescence images of hMSC-miR-3148 and hMSC-mcherry control cells ( \pm different concentration (7.8-125 nM) doxorubicin). Cells were stained with acridine orange/ethidium bromide to detect apoptotic and necrotic cells. White circles indicate resistant colonies.

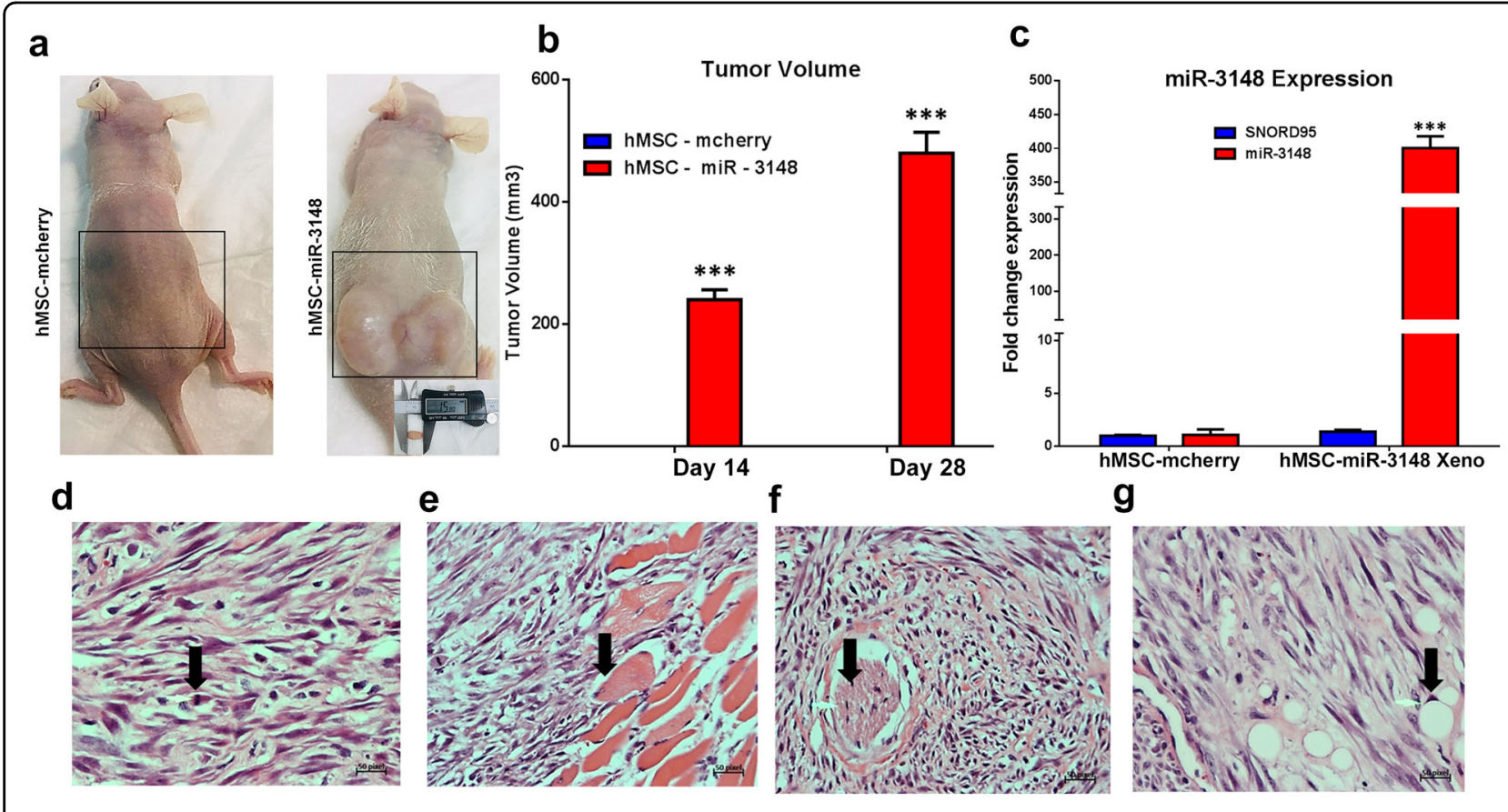

Fig. 5 The hMSC-miR-3148 cells exhibited enhanced tumor formation in vivo. Both hMSC-miR-3148 and hMSC-mcherry control cells were injected subcutaneously in nude mice. Representative images of tumor formed in each experimental group on day 28 is shown in (a). b Tumor size (log scale) in each experimental group on day 14 and day 28 is presented as bar chart. Data are presented as mean tumor volume \pm S.E.M., $n=4$; ${ }^{* * *} p<0.0005$. c qRT-PCR quantification of hsa-miR-3148 expression in hMSC-miR-3148 Xenograft tissue compared to mcherry control cells. Data are representative of three experiment and are presented as mean \pm S.D., $n=3$. $\mathbf{d}-\mathbf{g}$ Representative histopathological examination of hMSC-miR-3148 xenograft tumors using haematoxylin and eosin stain. Arrows indicate mitotic figures (d), muscle invasion (e), nerve surrounding (f), and adipocyte formation $(\mathbf{g})$.

sarcoma-like tumor tissue with numerous spindle and pleomorphic cells and with mitotic figures (Fig. 5d). The hMSC-miR-3148 tumors invaded skeletal muscles (Fig. 5e) and surrounding nerves (Fig. 5f). Interestingly, tumor tissue contained adipocytes suggesting a degree of adipocyte differentiation (Fig. 5g).

\section{miR-3148 regulates TGF $\beta$ signaling pathways through direct targeting of SMAD2}

In order to understand the molecular mechanism of miR-3148, we compared the list of downregulated transcripts in hMSC-miR-3148 cells to in silico predicted gene targets using the Targetscan database ${ }^{15}$ and targetscan algorithm $^{16}$. We identified 527 downregulated transcripts in hMSC-miR-3148 (Fig. 6a) and pathway analysis revealed significant inhibition of a number of canonical pathways related to osteogenesis, including TGF $\beta$ pathway (Fig. 6b). Interestingly, we observed several members of the SMAD family, which are TGF $\beta$ downstream effectors, to be significantly downregulated: SMAD2: -4.1 FC, SMAD6: -5.2 FC, and SMAD9: -4.4 FC (Supplementary Table 1). The expression of SMAD2 was subsequently validated using qRT-PCR and immunoblotting, given its key role in mediating the TGF $\beta$ signaling pathway. As seen in Fig. 6c, the expression and phosphorylation of SMAD2 were downregulated in hMSC-miR-3148. 


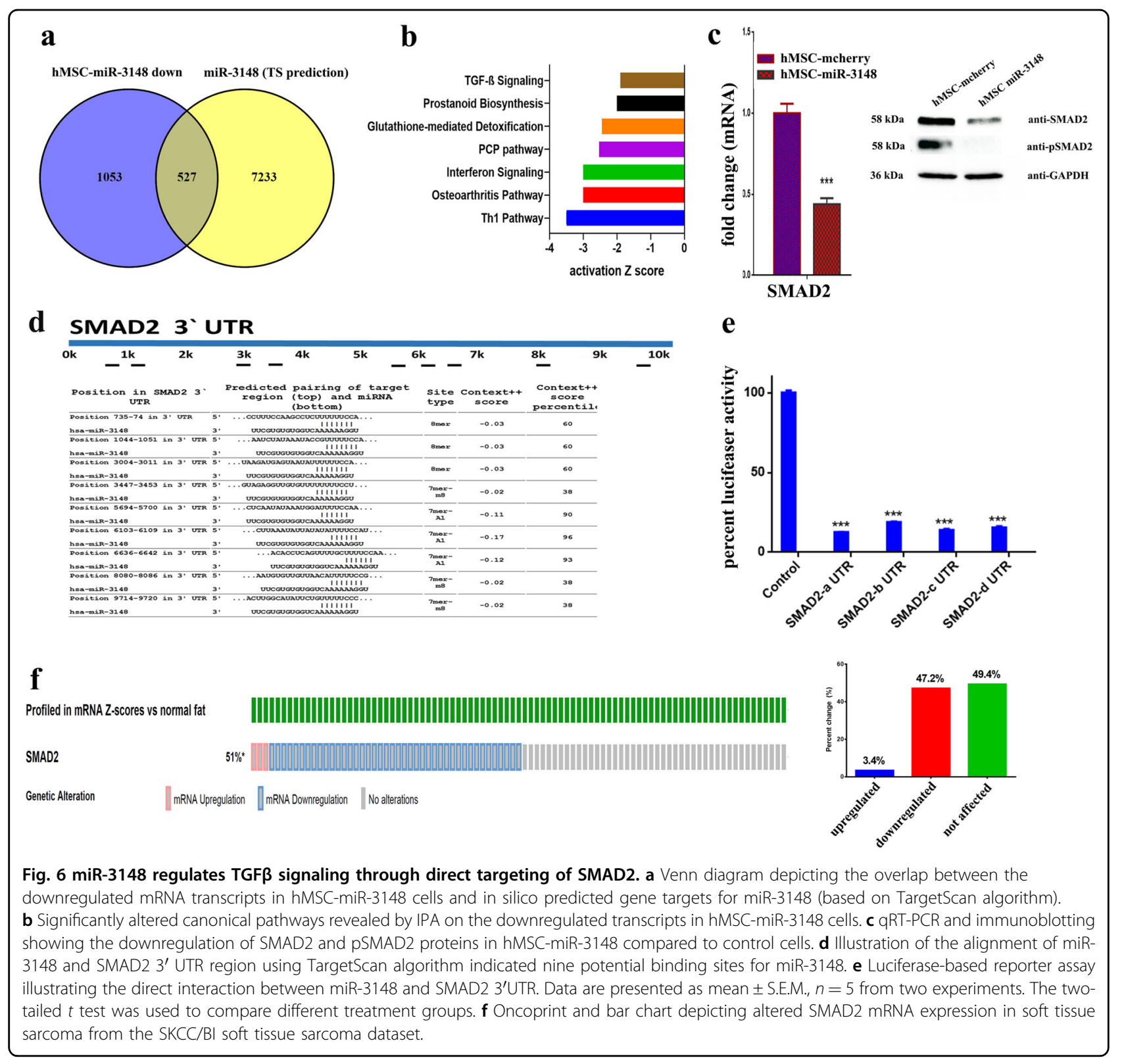

In silico analysis revealed nine potential binding sites for miR-3148 in SMAD2 3' UTR (Fig. 6d). Luciferase-based reporter assay corroborated our findings and validated SMAD2 as bone fide target for miR-3148 (Fig. 6e).

To highlight the clinical relevance of our findings, we examined the expression of SDMAD2 in the Memorial Sloan Kettering Cancer Center and Broad Institute (SKCC/BI) soft tissue sarcoma dataset, which revealed significant downregulation of SMAD2 in 47\% of sarcoma patients in these cohorts (Fig. 6f). In addition, higher expression of hsa-miR-3148 was associated with lower overall survival in multiple human cancer types (breast, cervical, esophageal, head and neck, ovarian, pancreatic, and uterine) and sarcoma (Fig. 7).

\section{Discussion}

miRNAs orchestrate complex regulatory networks across various biological systems. Each miRNA can regulate dozens of mRNA transcripts; and conversely, a single mRNA transcript can be regulated by several miRNAs. Interestingly, a single miRNA can exert multiple effects in various biological system. Dysregulated miRNA expression has been implicated in human diseases, including diabetes, cardiovascular, kidney diseases and cancer $^{17-19}$. For instance, the let-7 family of miRNAs regulates stem cell differentiation and predominantly exert a tumor suppressor effects ${ }^{20,21}$. Similarly, we have previously reported that miR-320 family plays a role in hMSC osteoblastic and adipocytic differentiation and to 


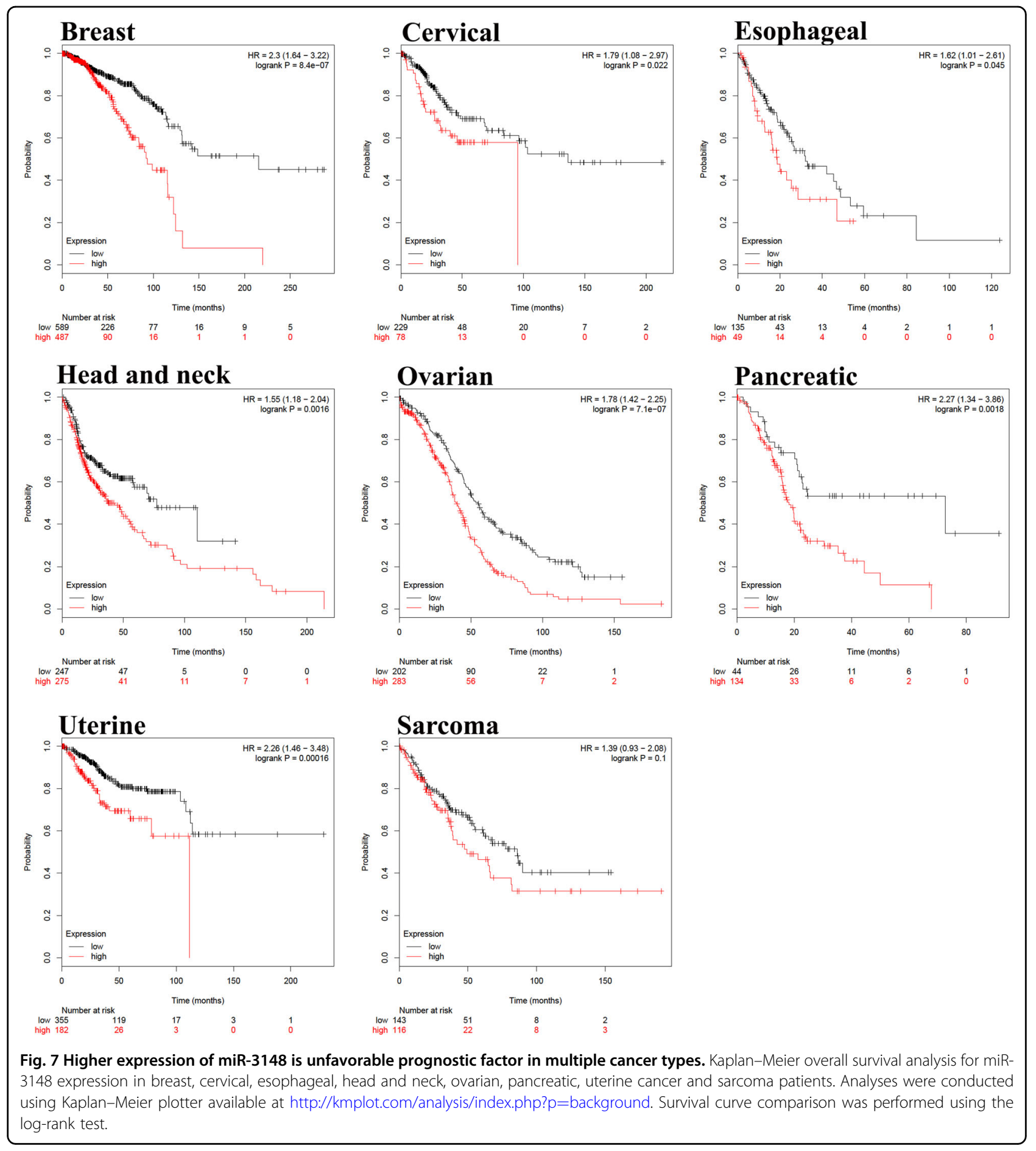

suppress colorectal cancer proliferation and migration $^{11,12}$. In the context of carcinogenesis, miRNA can be classified as oncomiRs or as tumor suppressor miRNAs. OncomiRs mediate their effects through downregulation of tumor suppressor genes, leading to abnormal cell functions and tumor development and progression ${ }^{22}$. For example, the miR-21 oncomiR is overexpressed in many human cancers, including, thyroid, breast, lung, CRC, pancreas, liver, gastric, cervical, skin, glioblastoma, and hematological malignancies ${ }^{23,24}$.

We observed that overexpression of miR-3148 in hMSC led to enhanced cell proliferation, induced a transformed cellular phenotype as well as formation of sarcoma-like tumors when implanting the cells in vivo. We have also 
observed that miR-3148 targets TGF $\beta$ signaling pathway. The role of TGF $\beta$ and BMPs is well documented during bone formation, which signals through both canonical SMAD-dependent and noncanonical SMAD-independent signal transduction pathways ${ }^{25}$. In the context of tumorigenesis, TGF $\beta$ pathway exerts opposing role either as promoter or as suppressor of tumor development and progression $^{26}$. Several studies highlighted an important role for TGF $\beta$ in regulating cell proliferation and promoting apoptosis in normal and transformed cells ${ }^{27}$. In breast and colon cancers, TGF $\beta$ signaling exerts antineoplastic effect during early stages, but eventually it promotes carcinogenesis through mediating epithelial mesenchymal transition (EMT) ${ }^{28}$. Interestingly, disruption of TGF $\beta$ signaling in the prostate of SV40 large T antigen transgenic animals, did not affect the size of neoplastic tumors, but instead it promoted tumor metastasis $^{29}$. Also, disruption of SMAD2 accelerates malignant progression of intestinal tumors in apc knockout mice, although the total number of intestinal tumors in those animals was not affected ${ }^{30}$. Taken together, the current studies suggest that disruption of TGF $\beta$ accelerates tumorigenesis, while the loss of TGF $\beta$ signaling is not sufficient to trigger malignant transformation. Our data are in agreement with the aforementioned reports where we observed miR-3148 to promote malignant transformation of the telomerized-hMSC cells, while forced expression of miR-3148 on its own has no significant effects on the growth of primary skin derived stromal cells (Supplementary Fig. 4). Telomerized-hMSC model employed in current study has normal karyotype, but exhibited deletion of the Ink4a/ARF locus and therefore is more prone to malignant transformation ${ }^{31}$, which provides a plausible explanation for the observed data of differential effects of miR-3148 on telomerizedhMSC compared to primary stromal cells. It also suggests that inactivation of TGF $\beta$ via miR-3148/SMAD2 axis is a "secondary hit" accelerating malignant transformation. In Ewing's sarcoma (ES), elevated expression of miR-20b was shown to directly target TGF $\beta$ receptor II (TGFBR2) leading to elevated MYC expression, hence inhibition of miR-20b reduced ES cell grow, cell cycle progression, and in vivo tumor formation, thus corroborating the important role of loss of TGF $\beta$ signaling in promoting ES tumor formation $^{32}$. In addition to the SMAD2/TGFB pathway, miR-3148-hMSC cells exhibited upregulation and downregulation of several onco- and tumor suppressor genes, respectively. The role of other genes in regulating hMSC differentiation and tumorigenesis in the context of miR3148 remains to be elucidated.

MiR-3148-hMSC cells exhibited enhanced resistance to doxorubicin, a commonly used chemotherapy in the treatment of human sarcomas. In order to provide potential mechanism by which miR-3148 confers doxorubicin resistance, gene expression data from GSE3362 was retrieved and the upregulated and downregulated genes in doxorubicin-resistant sarcoma were identified and were crossed with the list of differentially expressed genes in miR-3148-hMSC cells. Our data revealed common upregulated (ACADVL, RAG1, CYP51A1, PLAT, ITGA5, LOX, MAP4, MMP1, SCG5, STC1, THBS1, ZNF91, TNFRSF25, DDIT3, SLC16A3, SYNGR2, XRCC3, SEMA3A, POLR3G, TFPI2, KLF2, REEP2, TOR4A, STEAP3, APBB1IP, and SLC25A19) and common downregulated (CYP1B1, GJA1, IGFBP2, GSTM3, CD70, AMPH, LIF, MSX1, OAS2, THBS4, TPD52L1, BHLHE40, RUNX3, FGL1, DHRS3, FGR, SLC7A8, CD24, CELSR1, TCAF1, DACT1, PHF10, FEZ1, and C15orf48), Supplementary Fig. 6. For instance, RUNX3 was previously shown to inhibit hepatocellular carcinoma growth in vitro and in vivo in combination with Adriamycin ${ }^{33}$. Concordantly, reduced expression of AMPH1 was previously shown to be associated with breast cancer metastatic, advanced stage, poor clinical outcome, and Paclitaxel plus 5-fluorouracil/ epirubicin/ cyclophosphamide treatment resistance ${ }^{34}$. The role of other genes in mediating doxorubicin residence in miR3148-hMSC warrants further investigation.

A number of studies highlighted various mechanism leading to malignant transformation of hMSCs. For instance, methylation of HIC ZBTB Transcriptional Repressor 1 (HIC1) and Ras Association Domain Family Member 1 (RassF1A) tumor suppressor genes was sufficient to transform bone marrow MSCs into malignant cells endowed with tumor-initiating capabilities ${ }^{35}$. Alternatively, a number of genetic modification were utilized to transform hMSCs into malignant sarcoma cells. The combination of hTERT/H-RAS and hTERT/H-RAS/ SV40-LT has been demonstrated to induce pleomorphic sarcoma cells ${ }^{36-38}$. Transformed hMSCs using combination of hTERT and H-RAS retained their adipocytic and chondrogenic, while osteoblastic differentiation was completely lost ${ }^{38}$. Our current study corroborates these findings since miR-3148 overexpressing hMSC-TERT cells exhibited enhanced adipogenesis and suppressed osteogenesis, suggesting the loss of osteogenic differentiation is a common feature during malignant transformation of hMSC. Interestingly, we found positive correlation between elevated expression of miR-3148 and poor prognosis in several human cancer types, including sarcomas thus highlighting miR-3148 as a possible prognostic biomarker in various human cancers. In support of this hypothesis, recent data reported higher expression of miR-3148 in 3D-spheroids formed using the HCT116 human CRC model $^{39}$. miR-3148-overexpressing HCT116 cells exhibited enhanced tumor formation in vivo, were resistant to hypoxic conditions and exhibited higher sensitivity to MAPKK and ERK inhibitors ${ }^{39}$. 
In addition to its possible role in carcinogenesis, a role for miR-3148 in the context of other pathological conditions has been reported. miR-3148 levels were inversely correlated with Toll-like receptor 7 (TLR7) transcript levels in peripheral blood monocytes obtained from systemic lupus erythematosus (SLE) patients ${ }^{40}$. Interestingly, the G allele of rs3853839 in the 3' UTR of TLR7 has diminished binding capability to miR-3148, which was associated with elevated TLR7 transcript expression and increased risk for SLE, thus implicating miR-3148-TLR7 circuit in SLE pathogenesis ${ }^{40}$. Elevated levels of miR-3148 were also associated with congestive heart failure (CHF) in a cohort of $44 \mathrm{CHF}$ patients and 15 healthy subjects ${ }^{41}$. In addition, downregulation of miR-3148 was observed in chronic pulmonary hypertension ${ }^{42}$.

In conclusion, our data provide novel insight on the role of miR-3148-SMAD2-TGF $\beta$ axis during malignant transformation of hMSCs into sarcoma-like cancer and suggest therapeutic approaches targeting miR-3148/ SMAD2/TGF $\beta$ pathway as plausible therapeutic strategy.

\section{Materials and methods}

\section{Cell culture and lentiviral transduction}

We employed a well-characterized telomerase-transduced adult bone marrow hMSC cell line (hMSC-TERT) that expresses all known markers and possess similar differentiation potential to primary hMSCs in vitro and in vivo ${ }^{43,44}$. For simplicity, this line is referred to as hMSC throughout the paper. Those cells were cultured in Dulbecco's modified Eagle's medium (DMEM) supplemented with $4500 \mathrm{mg} / \mathrm{l} \mathrm{D}$-glucose, $4 \mathrm{mM} \mathrm{L}$-glutamine, $110 \mathrm{mg} / \mathrm{l}$ sodium pyruvate, $10 \%$ fetal bovine serum (FBS), $1 \%$ penicillin-streptomycin, and nonessential amino acids as previously described ${ }^{43}$.

Lentiviral particles encoding for hsa-miR-3148 (LPHmiR0781-MR03-0200-S) and control particles were purchased from Genecopoeia (Genecopoeia Inc., Rockville, MD, USA). Transduction was performed as previously described ${ }^{12}$. Briefly, hMSCs were treated with $20 \mu \mathrm{l}$ of crude lentiviral particles in $500 \mu \mathrm{l}$ of transduction medium (DMEM $+5 \%$ heat-inactivated serum and $1 \%$ Pen-Strep (Invitrogen)) with polybrene $(8 \mu \mathrm{g} / \mathrm{ml}$; Sigma, St. Louis, MO, USA). After $24 \mathrm{~h}$, media was removed and cells were selected with puromycin $(1 \mu \mathrm{g} / \mathrm{ml}$, Sigma, St. Louis, MO, USA) for 1 week until stably transduced cells were generated. Transduction efficiency was confirmed using qRT-PCR for miR-3148 expression compared to mcherry-transduced cells.

\section{Gene expression profiling by microarray}

Gene expression profiling was performed as previously described $^{45}$. In brief, total RNA was extracted using the total tissue RNA purification kit from Norgen-Biotek Corp. (Thorold, ON, Canada) and quantified in
NanoDrop 2000 spectrophotometer (Thermo Scientific, Wilmington, DE, USA). One-hundred fifty ng of total RNA was labeled and then hybridized to the Agilent Human SurePrint G3 Human GE $8 \times 60$ k microarray chip and subsequently data were analyzed using GeneSpring 13.0 software (Agilent Technologies, Palo Alto, CA, USA). Single experiment pathway analysis tool was used with twofold expression cutoff $(p<0.05)$. Benjamini-Hochberg False Discovery Rate (FDR) method was used for multiple testing corrections.

\section{Ingenuity pathway and network analysis}

Pathway analyses were conducted using Ingenuity pathway (Ingenuity Systems, http://www.ingenuity.com) ${ }^{46}$. Differentially expressed genes exhibiting $\geq 2 \leq$ and $p$ value $<$ 0.05 were subjected to core analysis using the human database. Enriched networks categories were algorithmically generated based on their connectivity and ranked according to the $Z$ score.

\section{qRT-PCR}

The expression levels of the indicated mRNA transcipts were validated using SYBR Green-based qRT-PCR using the applied biosystems $\mathrm{ViiA}^{\mathrm{TM}} 7$ real-time PCR system (ThermoFisher Scientific). Primers used in current study are listed in Table 1. Differential expression was calculated using the $2 \Delta \mathrm{CT}$ method and data were plotted as bar diagram.

\section{Protein extraction for mass spectrometry}

Proteins were extracted from three biological replicate $\left(\sim 2 \times 10^{6}\right.$ cells $)$ from the hMSC-miR-3148 or hMSC-

Table 1 List of SYBR green primers used in current study.

\begin{tabular}{|c|c|c|}
\hline No & Name & Sequence \\
\hline \multirow[t]{2}{*}{1} & AP2 & F 5' TGGTTGATITTCCATCCCAT \\
\hline & & R 5' GCCAGGAATTTGACGAAGTC \\
\hline \multirow[t]{2}{*}{2} & LEP & F 5' CAGCGGTTGCAAGGCCCAAGA \\
\hline & & R 5' GGCCAAAGCCACAAGAATCCGC \\
\hline \multirow[t]{2}{*}{3} & OC & F 5' GGCAGCGAGGTAGTGAAGAG \\
\hline & & R 5' CTCACACACCTCCCTCCTG \\
\hline \multirow[t]{2}{*}{4} & ON & F 5' GAGGAAACCGAAGAGGAGG \\
\hline & & R 5' GGGGTGTTGTTCTCATCCAG \\
\hline \multirow[t]{2}{*}{5} & ALP & F 5' GGAACTCCTGACCCTTGACC \\
\hline & & R 5' TCCTGTTCAGCTCGTACTGC \\
\hline \multirow[t]{2}{*}{6} & RUNX2 & F 5' GTAGATGGACCTCGGGAACC \\
\hline & & R 5' GAGGCGGTCAGAGAACAAAC \\
\hline \multirow[t]{2}{*}{7} & $\beta$-actin & F 5' AGCCATGTACGTTGCTA \\
\hline & & R 5' AGTCCGCCTAGAAGCA \\
\hline
\end{tabular}


mcherry cells directly in lysis buffer $(0.5 \mathrm{~mL}, \mathrm{pH} 8.8$, $30 \mathrm{mM}$ Tris buffer containing $7 \mathrm{M}$ urea, $2 \mathrm{M}$ thiourea, $2 \%$ Chaps, $1 \times$ protease inhibitor mix). The suspension was shaken for $1 \mathrm{~h}$ at room temperature and then sonicated (Microsonicator, Qsonica Sonicators, USA; 30\% pulse, two intervals of $1 \mathrm{~min}$ each, separated by a $1 \mathrm{~min}$ gap in ice). Fifty $\mathrm{mM}$ dithiothreitol was then added and the protein extracts were centrifuged $\left(20,000 \times g, 1 \mathrm{~h}, 4^{\circ} \mathrm{C}\right)$. The pellet was then removed and the solubilized proteins in the supernatant were precipitated using a 2D clean-up kit according to the manufacturer's protocol (GE Healthcare, USA).

\section{Protein labeling with cyanine dyes}

The protein pellets were solubilized in labeling buffer (7 M Urea, $2 \mathrm{M}$ Thiourea, $30 \mathrm{mM}$ Tris- $\mathrm{HCl}$, 4\% CHAPS, $\mathrm{pH}$ 8.5). Insoluble materials were pelleted by centrifugation $(12,000 \times g$, room temperature $(\mathrm{RT}), 5 \mathrm{~min})$, protein concentrations were determined in triplicate using the 2D-Quant kit (GE Healthcare, USA), and the $\mathrm{pH}$ of the samples was adjusted to 8.5 using $\mathrm{NaOH}(100 \mathrm{mM})$. Proteins were labeled ( 400 pmol of CyDye ${ }^{\mathrm{TM}}$ DIGE Fluor dyes, GE Healthcare, UK) in $1 \mu \mathrm{l}$ of DMF and then mixed with sample containing $50 \mu \mathrm{g}$ of protein. Samples were incubated on ice for $30 \mathrm{~min}$ in the dark. The labeling reaction was terminated by adding $1 \mu \mathrm{l}$ of $10 \mathrm{mM}$ lysine. Each sample was covalently labeled with a fluorophore, either Cy3 or Cy5. A mixture of equal amounts of protein isolated from each and every sample in the experiment was labeled with $\mathrm{Cy} 2$ and used as internal standard.

\section{Two dimensional gel electrophoresis (2D-DIGE)}

First dimension analytical gel electrophoresis was performed as follows. Six Immobiline Dry Strips $(24 \mathrm{~cm}, \mathrm{pH}$ 3-11; GE Healthcare, Sweden) were passively re-hydrated $(30 \mathrm{~V}, 12 \mathrm{~h})$. This was followed by isoelectric focusing using an Ettan IPGphor IEF unit (GE Healthcare, Sweden). Focusing was performed at $20^{\circ} \mathrm{C}$, at $50 \mu \mathrm{A}$ per strip, according to the following step and hold sequence: i.e., (1) $500 \mathrm{~V}$ for $1 \mathrm{~h}$, , (2) $1000 \mathrm{~V}$ for $1 \mathrm{~h}$, (3) $8000 \mathrm{~V}$ for $3 \mathrm{~h}$, (4) $45,000 \mathrm{~V}$ for $1 \mathrm{~h}$. After the first dimension, the strips were equilibrated and separated on 12.5\% (SDS-PAGE) gels using an Ettan Dalt Six device (GE Healthcare, Sweden). The gels were scanned with a Typhoon 9400 scanner (GE Healthcare) using appropriate wavelengths and filters for Cy2, Cy3, and Cy5 dyes. Total protein (1 mg) was obtained from a pool of equal protein amounts of each sample. This was denatured in lysis buffer, then mixed in a rehydration buffer. Gels were then stained by Colloidal Coomassie Blue staining (Supplementary Fig. 4).

\section{Protein Identification by MALDI-TOF MS}

The spots from Coomassie-stained gels were excised manually, washed, and digested according to a previously published protocol ${ }^{47}$. The mixture of tryptic peptides $(1 \mu \mathrm{L})$, derived from each protein, was spotted onto a MALDI target (384 anchorchip MTP $800 \mu \mathrm{m}$ Anchorchip; BrukerDaltonik, Germany) together with $0.8 \mu \mathrm{L}$ of matrix (10 mg $\alpha$-cyano-4-hydroxycinnamic acid (CHCA) in $1 \mu \mathrm{L}$ of $30 \% \mathrm{CH} 3 \mathrm{CN}$ and $0.1 \%$ aqueous $\mathrm{CF} 3 \mathrm{COOH}$ ) and then left to dry (RT) before MS analysis. Spectra were acquired using a MALDI-TOF MS (UltraFlexTrem, Bruker Daltonics) in the positive mode with target voltage of $25 \mathrm{kV}$ and pulsed ion extraction voltage of $20 \mathrm{kV}$. The reflector voltage was set to $21 \mathrm{kV}$ and detector voltage to $17 \mathrm{kV}$. PMF were calibrated against a standard (Peptide Calibration Standard II, Bruker Daltonics). The PMF were processed using the Flex AnalysisTM software (version 2.4, Bruker Daltonics). The MS data were interpreted using BioTools v3.2 (Bruker Daltonics), together with the MASCOT search algorithm (version 2.0.04 updated 09/ 05/2017; Matrix Science Ltd., UK). MASCOT search parameters were set as follows: fixed propionamide modification of cysteine, oxidation of methionine as variable modification, one missed cleavage site (such as in the case of incomplete trypsin hydrolysis), and a mass tolerance of $100 \mathrm{ppm}$. Identified proteins were accepted as correct if they showed a MASCOT score $>56$ and $p<$ 0.05 . Not all spots of interest could be identified because some proteins were low in abundance and did not yield a sufficiently intense mass of fingerprints; other spots were mixtures of multiple proteins.

\section{Image acquisition and statistical analysis}

DIGE images were analyzed using Progenesis Same Spots v3.3 software (Nonlinear Dynamics Ltd., UK). First, images were aligned. Prominent spots were used to manually assign; 45 vectors to digitized images within each gel and then the automatic vector tool was used to add additional vectors (390 total vectors), which were manually revised and edited for correction if necessary. These vectors were used to warp and align gel images with a reference image of one internal standard across and within each gel. Gel groups were established according to the experimental design and spot normalized volume (NV) was used to select statistically significant spots. The software calculated the NV of each spot on each gel from Cy3 (or Cy5) to Cy2 spot volume ratio. The software performs log transformation of the spot volumes to generate normally distributed data. Log normalized volume (LNV) was used to quantify differential expression. Independent direct comparisons were made between hMSC-miR-3148 and hMSC-mcherry cells and fold differences and $p$ values were calculated using one-way ANOVA. All spots were pre-filtered and manually checked before applying the statistical criteria (ANOVAtest, $p \leq 0.05$ and fold $\geq 1.5$ ). Normalized spot volumes, instead of spot intensities, were used in statistical 
processing. Only those spots that fulfilled the abovementioned statistical criteria were submitted for MS analysis.

\section{Protein network Pathway analysis}

Pathway analysis was carried out by importing the quantitative data into the IPA software (Ingenuity ${ }^{\circledR}$ Systems, http://www.ingenuity.com). This software aids in determining the functions and pathways that are most strongly associated with the protein list by overlaying the experimental expression data on networks constructed from published interactions.

\section{Cell differentiation}

Adipocytic and Osteoblastic differentiation were performed as previously described ${ }^{43,48}$. In brief, cells were cultured in basal medium till $70-80 \%$ confluence. Osteogenic induction medium consisting of DMEM containing $10 \% \mathrm{FBS}, 1 \% \mathrm{P} / \mathrm{S}, 50 \mu \mathrm{g} / \mathrm{mL}$ L-ascorbic acid (Wako Chemicals GmbH, Neuss, Germany), $10 \mathrm{mM}$ $\beta$-glycerophosphate (Sigma), and $10 \mathrm{nM}$ calcitriol $[(1 \alpha, 25-$ dihydroxy vitamin D3) (sigma)], $10 \mathrm{nM}$ dexamethasone (Sigma) was added. Adipogenic-induction mixture containing 10\% FBS, 10\% Horse Serum (Sigma), 1\% P/S, $100 \mathrm{nM}$ dexamethasone, $0.45 \mathrm{mM}$ isobutyl methyl xanthine [Sigma], $3 \mu \mathrm{g} / \mathrm{mL}$ insulin (Sigma), and $1 \mu \mathrm{M}$ Rosiglitazone [(BRL49653) (Novo Nordisk, Bagsvaerd, Denmark)] were added to adipogenic cultures. Both induction media were replaced every 3 days.

\section{Cytochemical assays}

Cytochemical analyses were performed to confirm the adipocyte and osteoblast lineage differentiation of hMSC as previously described ${ }^{48}$. In brief, all cytochemical images were taken using Carl Zeiss-Axio observer1 equipped with digital camera (Axiocam MRc5, Göttingen, Germany).

\section{Oil red-O staining for adipocytes}

Cells were washed in PBS, fixed in $4 \%$ formaldehyde and stained for $1 \mathrm{~h}$ at RT with filtered Oil red-O staining solution (prepared by dissolving $0.5 \mathrm{~g}$ Oil red-O powder in $60 \%$ isopropanol). Cells were rinsed with water and then photographed.

\section{Nile red staining of oil filled droplets}

Cells were grown in flat bottom 96-well black microplates (Corning, NY, USA) and after washing, cells were stained with nile red $(5 \mu \mathrm{g} / \mathrm{ml}$ in PBS), followed by an incubation for $10 \mathrm{~min}$ at RT then washed twice with PBS. Fluorescent signal was measured using SpectraMax/M5 fluorescence spectrophotometer plate reader (Molecular Devices Co., Sunnyvale, CA, USA) using bottom well-scan mode where nine readings were taken per well using Excitation $(485 \mathrm{~nm})$ and Emission $(572 \mathrm{~nm})$ wavelength.

\section{Alkaline phosphatase activity (ALP) staining and quantification}

We used the BioVision ALP activity colorimetric assay kit (BioVision, Inc., Milpitas, CA, USA) to quantify ALP activity. Cells were cultured in 6-well (for image analysis) and 96-well plates under normal or osteogenic induction conditions, on day 10 cells were washed with PBS and were fixed in $3.7 \%$ formaldehyde $+90 \%$ ethanol for $30 \mathrm{~s}$ at RT. Subsequently, fixative was removed and $50 \mu \mathrm{l}$ of pNPP (p-nitrophenyl phosphate) solution was added and incubated for $1 \mathrm{~h}$ in the dark at RT. Reaction was subsequently stopped by adding $20 \mu \mathrm{l}$ stop solution then measured and photographed.

\section{Alizarin Red S (ALZ) staining for mineralized matrix formation}

Cells were differentiated and stained on day 21. Twelve well plates were washed with PBS and then fixed with $4 \%$ paraformaldehyde for $15 \mathrm{~min}$ at RT. Subsequently, cells were washed in distilled water and stained with $2 \%$ Alizarin Red S Staining Kit (ScienceCell, Research Laboratories) for $30 \mathrm{~min}$ at RT. Subsequently, the dye was washed off with water then plates were photographed and staining intensity was quantified.

\section{Cell proliferation}

The proliferation of hMSC-mcherry and hMSC-miR3148 cells was determined using alamarBlue assay as previously described ${ }^{12}$. Briefly, 5000 cells were cultured in a 96-well plate and cell proliferation was measured at the indicated time points by adding 1:10 volume of the alamarBlue assay reagent and measuring fluorescence excitation and emission wavelengths of $530 \mathrm{~nm}$ and $590 \mathrm{~nm}$, respectively).

\section{Clonogenic assay}

The colony forming ability was determined using clonogenic assay as previously described ${ }^{12}$. Briefly, cells were seeded in 12-well plates at different dilution (1:2-1:32). Primary seeding density was $0.015 \times 10^{6}$ cells per well, and incubated at $37^{\circ} \mathrm{C}$ under $5 \% \mathrm{CO} 2$ for 5 days. The plates were then washed and stained with Diff-Quik stain set (Siemens Healthcare Diagnostics), and subsequently scanned colonies were observed under microscope.

\section{Sphere formation assay}

Multicellular tumor spheroids were produced in $60 \mathrm{~mm}$ low cell binding dishes (Nunc; ThermoFisher Scientific). The hMSC-mcherry and hMSC-miR-3148 cells were trypsinized from monolayers and transferred to the dishes. The formation of tumor spheroids was performed with 10,000 cells. On day 10, established spheroids were analyzed and stained with acridine orange and ethidium bromide. 


\section{Cell cycle analysis by flow cytometry}

Cell cycle analysis was performed as described earlier ${ }^{48}$, briefly, cell pellets were washed in PBS and re-suspended and stained in $500 \mu \mathrm{l}$ PBS supplemented $100 \mu \mathrm{g} / \mathrm{ml}$ RNAse A (Sigma, St. Louis, MO, USA) and $50 \mu \mathrm{g} / \mathrm{ml}$ of Propidium Iodine and were analyzed by Navious flow cytometer (Beckman Coulter, Miami, Florida, USA). Staining was detected in the fluorescence channel (FL3) and the data were analyzed using Kaluza software (Beckman Coulter).

\section{Scratch assay}

For the assessment of cell migration, confluent cells were maintained in standard medium and subsequently, scratches were made using a plastic micropipette tip (yellow tip; 20-200 $\mu \mathrm{L}$ ). After washing, the medium was replaced with fresh medium. Photographs of the wounded area were taken immediately $(0 \mathrm{~h})$ and after $20 \mathrm{~h}$ using phase-contrast microscopy.

\section{Migration assay}

Conventional and real-time measurement of cell migration was carried out as described earlier ${ }^{12}$. In brief, real time migration was executed using the xCELLigence RTCA DP device (ACEA Biosciences, San Diego, CA). Cells were starved for $24 \mathrm{~h}$ in $1 \%$ serum media, followed by seeding $0.08 \times 10^{6}$ cells per well in 16-well microelectronic sensor plate, two chamber transwell plates (CIMplate insert; ACEA Bioscience) containing the respective serum conditions. Media containing $10 \%$ serum (chemoattractant) and $1 \%$ serum (negative control) were added to the bottom wells. Similarly, for conventional migration, the $\mathrm{BD}$ transwell migration system with $8 \mu$ pore size was utilized. Inserts were placed in a 24-well plate, and $0.1 \times$ $10^{6}$ cells in $1 \%$ serum were added to the top of the chamber, and 10\% serum added to the bottom of the chamber. Eighteen hours later, inserts were fixed and stained with SIEMENNS DIFF-QUICK stain set (Siemens Healthcare Diagnostics).

\section{Invasion assay}

For invasion assay, we followed similar set up as in the migration assay, in addition, extracellular matrix (ECM) material-Matrigel was added on top of the transwell membrane. Matrigel was thawed on ice and then $50 \mu \mathrm{l}$ was added to a 24-well transwell insert and was allowed to solidify at $37^{\circ} \mathrm{C}$ for $30 \mathrm{~min}$ to form a thin gel layer. Cells were added on top of the Matrigel coating to simulate invasion through the ECM.

Acridine orange and ethidium bromide staining $(\mathrm{AO} / \mathrm{EtBr})$

$\mathrm{AO} / \mathrm{EtBr}$ stainig was performed as previously described; ${ }^{12}$ briefly, after doxorubicin drug treatment, both hMSC-mcherry and hMSC-miR-3148 cells were stained with dual fluorescent staining solution $(1 \mu \mathrm{l})$ containing $100 \mu \mathrm{g} / \mathrm{ml} \mathrm{AO}$ and $100 \mu \mathrm{g} / \mathrm{ml} \mathrm{EtBr}$ (Sigma, St. Louis, $\mathrm{MO})$. Cells were washed twice with PBS and were gently mixed with $\mathrm{AO} / \mathrm{EtBr}(1: 100)$ dye solution for $1 \mathrm{~min}$. The cells were then observed and photographed using a Nikon Eclipse Ti fluorescence microscope. Cells cultured without drug were considered as experiment control.

\section{Luciferase reporter assay}

Luciferase reporter assay was performed as previously described $^{12}$. Briefly, HEK293 cells were transfected with complexes containing control or SMAD2 UTR plasmids (100 ng), pre-miR control or pre-miR-3148 (50 nM) mixed with lipofectamine 2000 (Part No: 52758; Invitrogen) in Opti-MEM (11058-021; Gibco, Carlsbad, CA, USA). Luciferase activity was measured using the SecretePair $^{\mathrm{TM}}$ Dual Luminescence assay kit (Secrete Gaussia luciferase (GLU) and Secreted Alkaline Phosphatase (SEAP); GeneCopeia Inc., USA) according to the manufacturer's instructions luminescence was measured in SpectraMax M5 (Molecular Devices; USA) luminescence reader. The ratio of luminescence intensities of the GLU over SEAP was calculated and normalized to controls.

\section{Western blot analysis}

SMAD2 and pSMAD2 protein expression was analyzed by western blot as previously described ${ }^{48}$. Briefly, cell lysate samples containing $30 \mu \mathrm{g}$ of total protein were separated and blotted using the Bio-Rad V3 Western Workflow system according to the manufacturer's recommendation. Immunoblotting was performed using Phospho-SMAD2 (Ser465/Ser467), cat\# E8F3R Rabbit $\mathrm{mAb}$ and anti-SMAD2, cat\# D43B4 XP® Rabbit mAb, cell signaling technology (Danvers, MA, USA). The primary antibody was incubated overnight at $4{ }^{\circ} \mathrm{C}$. Horseradish peroxidase (HRP)-conjugated goat anti-rabbit was used as the secondary antibody, whereas HRP-conjugated antiGAPDH (glyceraldehyde-3-phosphate dehydrogenase) antibody (1:1000; Invitrogen) was used as a loading control. Chemiluminescent detection was performed using WesternSure Chemiluminescent Substrate (LI-COR, Lincoln, NE, USA).

\section{In vivo xenograft assay in nude mice}

In vivo tumor formation was carried out as previously described $^{49}$. Briefly, 6-8-week-old male immunodeficient nude mice were utilized in the xenograft experiments. Two million of hMSC-mcherry or hMSC-miR-3148 cells were mixed (1:1) with Matrigel (BD Biosciences, San Jose, CA, USA) and subcutaneously injected into both flanks of nude mice. Tumor mass was measured on day 14 and 28 using a caliper, and tumor volume was calculated using the formula: volume $=($ tumor length $\times$ width $\times$ width $) / 2$ as described before ${ }^{50}$. At the end of the experiment, 
tumors were excised, fixed in $10 \%$ buffered formalin, embedded in paraffin, and sectioned on a microtome. Sections were then stained with hematoxylin/eosin and anti-Pan Keratin (AE1/AE3/PCK26).

\section{Ethical approval}

Animal experiments received the appropriate institutional ethical from the Animal Care Committees of King Saud University (No. KSU-SE-18-2).

\section{Immunohistochemistry}

Immunohistochemical (IHC) staining protocol was performed as previously described ${ }^{49}$. Four-micron-thick sections were stained using hematoxylin and eosin $(\mathrm{H}$ \& E) using the IHC staining protocol with fully automated IHC/ISH staining instrument (Ventana Medical system Inc, Tucson, Arizona, USA).

\section{SMAD2 expression in soft tissue sarcoma and miR-3148 pan-cancer survival analysis}

The expression of SMAD2 was interrogated in the (SKCC/BI soft tissue sarcoma dataset as described before $^{51}$. Prognostic value of miR-3148 in breast, cervical, esophageal, head and neck, ovarian, pancreatic, and uterine cancer and sarcoma was investigated as described before $^{52}$.

\section{Statistical analysis}

Statistical analyses and graph generation were performed using Microsoft Excel 2010 and GraphPad Prism 6.0 software (GraphPad, San Diego, CA, USA). $p$ values were calculated using the two-tailed $t$ test. $p$ value $<0.05$ was considered significant.

\section{Acknowledgements}

This work was supported by the Deanship of Scientific Research at King Saud University Research Group No. RG-1438-033. The publication of this article was funded by a start-up grant for Dr. Nehad Alajez (QB13) from Qatar Biomedical Research Institute (QBRI).

\section{Author details \\ 'Cancer Research Center, Qatar Biomedical Research Institute (QBRI), Hamad Bin Khalifa University (HBKU), Qatar Foundation (QF), Doha, Qatar. ${ }^{2}$ Stem Cell Unit, Department of Anatomy, College of Medicine, King Saud University, Riyadh, Saudi Arabia. ${ }^{3}$ Molecular Endocrinology Unit (KMEB), Department of Endocrinology, University Hospital of Odense and University of Southern Denmark, Odense, Denmark. ${ }^{4}$ Department of Pathology, King Saud University Medical City, Riyadh, Saudi Arabia. ${ }^{5}$ McGill University Health Centre and RI- MUHC, Montreal, QC, Canada. ${ }^{6}$ Departement of Medicine, University of Montreal, Montreal, QC, Canada. ${ }^{7}$ Proteomics Resource Unit, Obesity Research Center, College of Medicine, King Saud University, Riyadh 11461, Saudi Arabia. ${ }^{8}$ The National Center for Biotechnology (NCBT), Life Science and Environment Research Institute, King Abdulaziz City for Science and Technology (KACST), Riyadh, Saudi Arabia. ${ }^{9}$ Department of Cellular and Molecular Medicine, Danish Stem Cell Center (DanStem), University of Copenhagen, 2200 Copenhagen, Denmark}

\section{Author contributions}

R.V.: Data acquisition and paper writing; R.E., M.M., D.A., A.A.-R., D.H., R.H., H.B., A. M., I.O.A., and A.A.A.: Data acquisition and analysis; M.A., A.A., M.K., and N.M.A.: study design; N.M.A.: Acquisition of funding, data analysis, editing and final approval of the paper.

\section{Conflict of interest}

The authors declare that they have no conflict of interest.

\section{Publisher's note}

Springer Nature remains neutral with regard to jurisdictional claims in published maps and institutional affiliations.

The online version of this article (https://doi.org/10.1038/s41420-020-00312-z) contains supplementary material, which is available to authorized users.

Received: 18 February 2020 Revised: 14 June 2020 Accepted: 4 August 2020

Published online: 01 September 2020

\section{References}

1. Bushati, N. \& Cohen, S. M. microRNA functions. Annu. Rev. Cell Dev. Biol. 23 175-205 (2007)

2. Calin, G. A. \& Croce, C. M. MicroRNA-cancer connection: the beginning of a new tale. Cancer Res. 66, 7390-7394 (2006).

3. Iorio, M. V. et al. MicroRNA gene expression deregulation in human breast cancer. Cancer Res. 65, 7065-7070 (2005).

4. Vishnubalaji, R. et al. Genome-wide mRNA and miRNA expression profiling reveal multiple regulatory networks in colorectal cancer. Cell Death Dis. 6, e1614 (2015)

5. Ueda, T. et al. Relation between microRNA expression and progression and prognosis of gastric cancer: a microRNA expression analysis. Lancet Oncol. 11, 136-146 (2010).

6. Yanaihara, N. et al. Unique microRNA molecular profiles in lung cancer diagnosis and prognosis. Cancer Cell 9, 189-198 (2006).

7. Thayanithy, V. et al. Perturbation of 14q32 miRNAs-cMYC gene network in osteosarcoma. Bone 50, 171-181 (2012).

8. Drury, R., Verghese, E. T. \& Hughes, T. A. The roles of microRNAs in sarcomas. J. Pathol. 227, 385-391 (2012).

9. Hur, K. et al. MicroRNA-200c modulates epithelial-to-mesenchymal transition (EMT) in human colorectal cancer metastasis. Gut 62, 1315-1326 (2013).

10. Tay, Y., Zhang, J., Thomson, A. M., Lim, B. \& Rigoutsos, I. MicroRNAs to Nanog, Oct4 and Sox2 coding regions modulate embryonic stem cell differentiation. Nature 455, 1124-1128 (2008).

11. Hamam, D. et al. microRNA-320/RUNX2 axis regulates adipocytic differentiation of human mesenchymal (skeletal) stem cells. Cell Death Dis. 5, e1499 (2014).

12. Vishnubalaij, R. et al. MicroRNA-320 suppresses colorectal cancer by targeting SOX4, FOXM1, and FOXQ1. Oncotarget 7, 35789-35802 (2016).

13. Shimono, Y. et al. Downregulation of miRNA-200c links breast cancer stem cells with normal stem cells. Cell 138, 592-603 (2009).

14. Edmonson, J. H. et al. Randomized comparison of doxorubicin alone versus ifosfamide plus doxorubicin or mitomycin, doxorubicin, and cisplatin against advanced soft tissue sarcomas. J. Clin. Oncol. 11, 1269-1275 (1993).

15. Alajez, N. M. et al. MiR-218 suppresses nasopharyngeal cancer progression through downregulation of survivin and the SLIT2-ROBO1 pathway. Cancer Res. 71, 2381-2391 (2011).

16. Lewis, B. P., Burge, C. B. \& Bartel, D. P. Conserved seed pairing, often flanked by adenosines, indicates that thousands of human genes are microRNA targets. Cell 120, 15-20 (2005).

17. Paul, P. et al. Interplay between miRNAs and human diseases. J. Cell. Physiol. 233, 2007-2018 (2018).

18. Lim, H. J. \& Yang, J. L. Regulatory roles and therapeutic potential of microRNA in sarcoma. Crit. Rev. Oncol/Hematol. 97, 118-130 (2016).

19. Hamam, D., Ali, D., Kassem, M., Aldahmash, A. \& Alajez, N. M. microRNAs as regulators of adipogenic differentiation of mesenchymal stem cells. Stem Cells Dev. 24, 417-425 (2015).

20. Rybak, A. et al. A feedback loop comprising lin-28 and let-7 controls pre-let-7 maturation during neural stem-cell commitment. Nat. Cell Biol. 10, 987-993 (2008).

21. Boyerinas, B., Park, S. M., Hau, A., Murmann, A. E. \& Peter, M. E. The role of let-7 in cell differentiation and cancer. Endocr-Relat. Cancer 17, F19-F36 (2010). 
22. Shenouda, S. K. \& Alahari, S. K. MicroRNA function in cancer: oncogene or a tumor suppressor? Cancer Metastasis Rev. 28, 369-378 (2009).

23. Nguyen, D. D., Chang, S. Development of novel therapeutic agents by inhibition of oncogenic MicroRNAs. Int. J. Mol. Sci. 19 1-17 (2017).

24. Hamam, R. et al. Circulating microRNAs in breast cancer: novel diagnostic and prognostic biomarkers. Cell Death Dis. 8, e3045 (2017).

25. Wu, M., Chen, G. \& Li, Y. P. TGF-beta and BMP signaling in osteoblast, skeletal development, and bone formation, homeostasis and disease. Bone Res. 4, 16009 (2016).

26. Akhurst, R. J. \& Derynck, R. TGF-beta signaling in cancer-a double-edged sword. Trends Cell Biol. 11, S44-S51 (2001).

27. Schuster, N. \& Krieglstein, K. Mechanisms of TGF-beta-mediated apoptosis. Cell Tissue Res. 307, 1-14 (2002).

28. Heldin, C. H., Vanlandewijck, M. \& Moustakas, A. Regulation of EMT by TGFbeta in cancer. FEBS Lett. 586, 1959-1970 (2012).

29. Tu, W. H. et al. The loss of TGF-beta signaling promotes prostate cancer metastasis. Neoplasia 5, 267-277 (2003).

30. Hamamoto, T. et al. Compound disruption of smad2 accelerates malignant progression of intestinal tumors in apc knockout mice. Cancer Res. 62 , 5955-5961 (2002).

31. Serakinci, N. et al. Adult human mesenchymal stem cell as a target for neoplastic transformation. Oncogene 23, 5095-5098 (2004).

32. Kawano, M., Tanaka, K., Itonaga, I., Iwasaki, T. \& Tsumura, H. MicroRNA-20b promotes cell proliferation via targeting of TGF-beta receptor II and upregulates MYC expression in Ewing's sarcoma cells. Int. J. Oncol. 51, 1842-1850 (2017).

33. Li, X. et al. RUNX3 inhibits growth of HCC cells and HCC xenografts in mice in combination with adriamycin. Cancer Biol. Ther. 7, 669-676 (2008).

34. Tokunaga, S. et al. Neoadjuvant chemotherapy with Nab-paclitaxel PLus Trastuzumab Followed by 5-fluorouracil/epirubicin/cyclophosphamide for HER2-positive operable breast cancer: a multicenter phase II trial. Anticancer Res. 39, 2053-2059 (2019).

35. Teng, I. W. et al. Targeted methylation of two tumor suppressor genes is sufficient to transform mesenchymal stem cells into cancer stem/initiating cells. Cancer Res. 71, 4653-4663 (2011).

36. Shima, $Y$. et al. In vitro transformation of mesenchymal stem cells by oncogenic H-rasVal12. Biochem. Biophys. Res. Commun. 353, 60-66 (2007).

37. Li, N. et al. Genetically transforming human mesenchymal stem cells to sarcomas: changes in cellular phenotype and multilineage differentiation potential. Cancer 115, 4795-4806 (2009).
38. Rodriguez, R., Rubio, R. \& Menendez, P. Modeling sarcomagenesis using multipotent mesenchymal stem cells. Cell Res. 22, 62-77 (2012).

39. Akamine, T. et al. miR-3148 is a novel onco-microRNA that potentiates tumor growth in vivo. Anticancer Res. 38, 5693-5701 (2018).

40. Deng, Y. et al. MicroRNA-3148 modulates allelic expression of toll-like receptor 7 variant associated with systemic lupus erythematosus. PLoS Genet. 9 e1003336 (2013).

41. Cakmak, H. A. et al. The prognostic value of circulating microRNAs in heart failure: preliminary results from a genome-wide expression study. J. Cardiovasc. Med. 16, 431-437 (2015).

42. Miao, R. et al. Microarray analysis and detection of MicroRNAs associated with chronic thromboembolic pulmonary hypertension. BioMed. Res. Int. 2017 8529796 (2017)

43. Vishnubalaji, R. et al. In vitro differentiation of human skin-derived multipotent stromal cells into putative endothelial-like cells. BMC Dev. Biol. 12, 7 (2012).

44. AIMuraikhi, N. et al. Stem cell library screen identified ruxolitinib as regulator of osteoblastic differentiation of human skeletal stem cells. Stem Cell Res. Ther. $\mathbf{9}$, 319 (2018).

45. Vishnubalaji, R. et al. Molecular profiling of ALDH1(+) colorectal cancer stem cells reveals preferential activation of MAPK, FAK, and oxidative stress prosurvival signalling pathways. Oncotarget 9, 13551-13564 (2018).

46. Calvano, S. E. et al. A network-based analysis of systemic inflammation in humans. Nature 437, 1032-1037 (2005).

47. Alfadda, A. A. et al. Proteomic analysis of mature adipocytes from obese patients in relation to aging. Exp. Gerontol. 48, 1196-1203 (2013).

48. Ali, D. et al. CUDC-907 promotes bone marrow adipocytic differentiation through inhibition of histone deacetylase and regulation of cell cycle. Stem Cells Dev. 26, 353-362 (2017).

49. Vishnubalaji, R. et al. Bone morphogenetic protein 2 (BMP2) induces growth suppression and enhances chemosensitivity of human colon cancer cells. Cancer Cell Int. 16, 77 (2016).

50. Alajez, N. M. et al. Efficacy of systemically administered mutant vesicular stomatitis virus (VSVDelta51) combined with radiation for nasopharyngeal carcinoma. Clin. Cancer Res. 14, 4891-4897 (2008).

51. Alajez, N. M. Large-scale analysis of gene expression data reveals a novel gene expression signature associated with colorectal cancer distant recurrence. PLoS ONE 11, e0167455 (2016).

52. Gyorffy, B. et al. An online survival analysis tool to rapidly assess the effect of 22,277 genes on breast cancer prognosis using microarray data of 1,809 patients. Breast Cancer Res. Treat. 123, 725-731 (2010). 